\title{
On the Computation of Solutions of Boundary Value Problems on Infinite Intervals
}

\author{
By R. M. M. Mattheij
}

\begin{abstract}
For solutions of linear boundary value problems defined on $[0, \infty)$ one has to study the stable or bounded solution manifold. A characterization of these manifolds is investigated here. A multiple shooting type algorithm is then developed to compute such solutions. This algorithm is fully adaptive and also covers problems where the ODE matrix does not tend to a limit (as is usually assumed), if the unstable manifold consists only of exponentially growing solutions. If the latter manifold also contains polynomially growing solutions, an extrapolation type approach is suggested. The theory is illustrated by a number of examples.
\end{abstract}

\section{Introduction. Consider the ODE}

$$
\frac{d x(t)}{d t}=L(t) x(t)+f(t), \quad 0 \leqslant t<\infty,
$$

where $L(t)$ is a continuous $n \times n$ matrix-valued function and $x(t)$ and $f(t)$ are $n$ vector-valued functions. Assume we have a boundary condition (BC)

$$
M_{0} x(0)+\lim _{\beta \rightarrow \infty} M_{1} x(\beta)=b .
$$

Quite often, (1.2) is not sufficient to define a unique solution to (1.1), (1.2), and an extra requirement

$$
\varlimsup_{\beta \rightarrow \infty}\|x(\beta)\| \text { exists }
$$

is needed. In fact, (1.3) restricts the set of potential solutions to the bounded solution manifold $\left(\|\cdot\|\right.$ denotes some norm on $\left.\mathbf{R}^{n}\right)$.

Boundary value problems (BVP) on infinite intervals have been studied extensively. Numerically, the usual approach is to look for a finite interval problem such that its solution approximates $x$ reasonably well on (a part of) that finite interval. Hence, the two main problems are: where to choose the "terminal point" $\beta$; and what $\mathrm{BC}$ to define at $\beta$. In cases where $L(t)$ approaches a limit, this can be investigated by studying the eigenvalues of the limit matrix. The terminal point should be chosen such that, e.g., the modes corresponding to the eigenvalues with positive real part have grown sufficiently; at the same time, natural BC's are induced by the directions of the complementary eigenvectors (or principal vectors), see [5], [6], [8], [9], [20].

Received March 25, 1985; revised January 31, 1986 and April 3, 1986.

1980 Mathematics Subject Classification. Primary 65L10.

Present address: Faculteit Wiskunde en Informatica, Technische Universiteit, Postbus 513, $5600 \mathrm{MB}$ Eindhoven, The Netherlands. 
Sometimes it is possible to use special techniques for second-order scalar problems, as in [20], double shooting as in [1], or a least-squares approach (which should work also in more general situations), as in [7].

A first problem is that of rotational activity of the fundamental solution (cf. [21]); thus it may be that the solution $x$ approaches a limit, but $L(t)$ does not. Obviously, the above-mentioned asymptotic approach then does not work. Secondly, from a computational point of view, it is important to be able to find a suitable terminal point $\beta$ automatically, without an elaborate preliminary analysis. Finally, if for example $L(t)$ and $f(t)$ are asymptotically constant, there may be modes that grow, but only polynomially (defective zero eigenvalues). This requires a fairly detailed analysis of the bounded solution space. In the present paper we try to address these three questions. If we only have exponentially growing homogeneous modes, then the algorithm given below gives a satisfactory solution to both the problem of possible rotational activity of these modes and also to the question where to choose terminal points. In fact, the method just computes a sufficient approximation to the bounded solution manifold. Because of the latter capability it can also be adapted to employ extrapolation techniques for iterative refinement of the solution in the case where we have polynomial (instead of exponential) growth. In that sense it is possible to treat the defective cases in the same way as elsewhere (e.g., [8]).

The paper is organized as follows. In Section 2 we consider the existence of solutions and in Section 3 their uniqueness within the bounded solution manifold. Then in Section 4 we describe an algorithm, based on multiple shooting, for computing a bounded solution within a prescribed accuracy on a prescribed interval. Cases where some of the unstable modes are growing only polynomially are treated in Section 5. In Section 6 we show how the algorithm determines the dimension of the unstable manifold and also what is done when there is no unique solution. Finally, a number of numerical examples are given in Section 7.

\section{Existence of Solutions.}

2.1. The Solution Space. Since our main objective is to describe an algorithm, we shall not go into depth with regard to existence questions. Besides, the subject is quite well developed; cf., e.g., [5], [6], [8], [9]. Our interest is mainly in problems where the solution space can be split into subspaces whose members have a certain growth behavior. Therefore, we shall use the following generalization of the dichotomy concept:

Assumption 2.1. Let $Y$ be a fundamental solution of (1.1) and $P_{1}, P_{2}, P_{3}$, and $P_{4}$ be projection matrices of rank $k_{1}, k_{2}, k_{3}$, and $k_{4}$, respectively, with

$$
P_{1}+P_{2}+P_{3}+P_{4}=I \quad\left(k_{1}+k_{2}+k_{3}+k_{4}=n\right)
$$

let $\lambda, \mu$ be negative numbers and, finally, $\kappa$ a positive number such that

(i) $\left\|Y(t) P_{1} Y^{-1}(s)\right\| \leqslant \kappa \exp \lambda(s-t), t<s$,

(ii) $\left\|Y(t) P_{2} Y^{-1}(s)\right\| \leqslant \kappa, t<s$,

(iii) $\left\|Y(t) P_{3} Y^{-1}(s)\right\| \leqslant \kappa, t>s$,

(iv) $\left\|Y(t) P_{4} Y^{-1}(s)\right\| \leqslant \kappa \exp \mu(t-s), t>s$.

The projection $P_{j}$ introduces a solution subspace $\mathscr{S}_{j}:=\left\{Y(t) P_{j} c \mid c \in \mathbf{R}^{n}\right\}, j=$ $1, \ldots, n$. The projections in 2.1 then have the following meaning: $\mathscr{S}_{1}$ consists of solutions that increase exponentially. $\mathscr{S}_{2}$ consists of solutions that do not decrease. 
If $P_{1}$ is chosen such that $k_{1}$ is as large as possible, $\mathscr{S}_{2}$ may still have polynomially increasing solutions. $\mathscr{S}_{3}$ consists of solutions that do not increase. Finally, $\mathscr{S}_{4}$ consists of exponentially decreasing solutions. If $P_{4}$ is chosen such that $k_{4}$ is as large as possible, then the solutions in $\mathscr{S}_{3}$ do not increase or decrease. Although the splitting in 2.1 covers much more general situations, it is useful to think of an autonomous ODE, which has $k_{1}$ eigenvalues with strictly positive real part, $k_{2}+k_{3}$ eigenvalues with zero real part and such that their geometric multiplicity (= number of genuine independent eigenvectors) is at most $k_{3}$, and finally $k_{4}$ eigenvalues with negative real part.

In order to further simplify our analysis, it is useful to note that the projection $P_{j}$ can be written as

$$
P_{j}=H^{j}\left[H^{j}\right]^{T},
$$

where $H^{j}$ consists of $k_{j}$ orthonormal columns. Writing

$$
H:=\left(H^{1}\left|H^{2}\right| H^{3} \mid H^{4}\right),
$$

an orthogonal matrix, we immediately find that

$$
Z(t):=Y(t) H=\left(Z^{1}(t)\left|Z^{2}(t)\right| Z^{3}(t) \mid Z^{4}(t)\right)
$$

is a fundamental solution with similar properties as those mentioned in 2.1 , but with projection matrices $\hat{P}_{j}$ such that $\hat{P}_{j}$ is a zero matrix, except for the diagonal entries with indices $\left(\sum_{l=1}^{j-1} k_{l}\right)+1, \ldots, \sum_{l=1}^{j} k_{l}$, which are 1 . From now on we shall assume that $Y$ already has a form like $Z$, so that the projections simply amount to picking the appropriate column vectors of $Y$. Moreover, we tacitly assume that $P_{1}$ is chosen such that $k_{1}$ is maximal.

Under suitable circumstances there exists a bounded solution $y_{b}$ of the problem (1.1), for which (cf. [2, Chapter 4])

$$
\begin{aligned}
y_{b}(t)= & \int_{0}^{t} Y(t)\left(P_{3}+P_{4}\right) Y^{-1}(s) f(s) d s \\
& +\int_{t}^{\infty} Y(t)\left(P_{1}+P_{2}\right) Y^{-1}(s) f(s) d s .
\end{aligned}
$$

Alternatively,

$$
\int_{0}^{t} Y(t) P_{3} Y^{-1}(s) f(s) d s
$$

may sometimes be replaced by

$$
\int_{t}^{\infty} Y(t) P_{3} Y^{-1}(s) f(s) d s .
$$

Henceforth, we shall simply assume that such a bounded solution exists (and do not go into the requirements this imposes on $f$ ).

2.2. Existence of the Solution to (1.1), (1.2). In order to have a bounded solution to (1.1), (1.2), we must exclude components of $\mathscr{S}_{1}$ and $\mathscr{S}_{2}$ from $x$. Hence, $x \in y_{b} \oplus$ $\mathscr{S}_{3} \oplus \mathscr{S}_{4}$. As was shown by a simple example in [17], this imposes several restrictions on $M_{1}$ in those cases where the "directions" of modes in $\mathscr{S}_{1}, \mathscr{S}_{2}$ do not tend to a limit. Of course, problems of this kind are avoided if $M_{1}=0$. Below we shall give slightly more general conditions for the existence of such a conditionally stable 
solution $x$. If $c \in \mathbf{R}^{n}$ is such that

$$
x(t)=y_{b}(t)+Y(t)\left(P_{3}+P_{4}\right) c
$$

then

$$
\begin{aligned}
M_{0} y_{b}(0)+\lim _{\beta \rightarrow \infty} M_{1} y_{b}(\beta)+M_{0} Y(0)\left(P_{3}+P_{4}\right) c & \\
& +\lim _{\beta \rightarrow \infty} M_{1} Y(\beta)\left(P_{3}+P_{4}\right) c=b,
\end{aligned}
$$

provided the limits exist. This yields

Property 2.7. The BVP (1.1), (1.2) has a bounded solution if

$$
\lim _{\beta \rightarrow \infty} M_{1} Y(\beta)\left(P_{3}+P_{4}\right)
$$

exists.

We shall henceforth explicitly exclude cases where $\lim _{\beta \rightarrow \infty} y_{b}(\beta)$ does not exist. Next, we give the following (simply verifiable) sufficient conditions for $x$ to exist.

Property 2.8. $\lim _{\beta \rightarrow \infty} M_{1} Y(\beta)\left(P_{3}+P_{4}\right)$ exists, if

(i) $M_{1}=0$ or

(ii) $P_{3}=0$ or

(iii) $\lim _{\beta \rightarrow \infty} M_{1} Y(\beta) P_{3}$ exists.

Theoretically, we need the existence of $\lim _{\beta \rightarrow \infty} M_{1} Y(\beta)\left(P_{3}+P_{4}\right) c$ only for some specific vector $c$. However, if we change $b$ (in (1.2)) slightly, the value of $c$ will change accordingly; hence in order to make the result practically relevant, it should be independent of $c$.

\section{Uniqueness of Solutions.}

3.1. The Number of Independent BC's. As we saw before, modes of $\mathscr{S}_{1}$ and $\mathscr{S}_{2}$ did not play a role in determining $x$, since $x \in y_{b} \oplus \mathscr{S}_{3} \oplus \mathscr{S}_{4}$. In order to have a unique solution of the BVP, we thus have to require that $M_{0}$ and $M_{1}$ provide for $k_{3}+k_{4}$ independent relations, hence

Property 3.1. If the problem has a solution $x$, then $x$ is unique if

$$
m:=\operatorname{rank}\left(\left[M_{0} Y(0)+M_{1} Y(\beta)\right]\left(P_{3}+P_{4}\right)\right)=k_{3}+k_{4}
$$

for all $\beta$ sufficiently large.

Since there might be some arbitrariness in the choice of $P_{2}$ and $P_{3}$, the above statement should be read in the proper spirit. To be more specific, if $\mathscr{S}_{2}$ consists of solutions that increase as $t \rightarrow \infty$, then 3.1 says something about the bounded solution. However, if $\mathscr{S}_{2}$ contains some nongrowing solutions as well, then uniqueness is meant relative to the subspace $\mathscr{S}_{2} \oplus \mathscr{S}_{3}$. Of course, from a practical point of view, the latter result is not very useful if no detailed information about these subspaces is available. Hence

COROLlaRY 3.2. If $P_{3}$ is chosen such that $k_{3}$ is maximal, then there exists a $\left(k_{3}+k_{4}-m\right)$-dimensional manifold of bounded solutions, where $m$ is defined in Property 3.1.

3.2. Conditioning of the Problem. The vector $c$ in (2.6b), at a first glance, satisfies an underdetermined system. However, if we consider that the vector we are interested in is $\left(P_{3}+P_{4}\right) c$, i.e., the vector of the last $k_{3}+k_{4}$ coordinates, it follows that 
it must solve the (rank $m$ ) linear system

$$
R c=\tilde{b}:=b-M_{0} y_{b}(0)-\lim _{\beta \rightarrow \infty} M_{1} y_{b}(\beta),
$$

where

$$
R:=\lim _{\beta \rightarrow \infty} R(\beta):=\lim _{\beta \rightarrow \infty}\left[M_{0} Y(0)+M_{1} Y(\beta)\right]\left(P_{3}+P_{4}\right) .
$$

Similar to the conditioning bound that was given in [15], we may now introduce

$$
\mathscr{C} \mathcal{N}(\beta):=\max _{0 \leqslant t \leqslant \beta}\left\|Y(t)\left(P_{3}+P_{4}\right) R^{+}(\beta)\right\|,
$$

where $R^{+}(\beta)$ is the pseudo-inverse of $R(\beta)$; cf. [22]. On account of Corollary 3.5 in [22] we deduce

Property 3.4. $\lim _{\beta \rightarrow \infty} \mathscr{C} \mathcal{N}(\beta) \equiv: \mathscr{C} \mathscr{N}$ exists, if $m=k_{3}+k_{4}$, where $m$ is defined in Property 3.1.

Since $Y(t)\left(P_{3}+P_{4}\right)$ represents bounded modes only, we may estimate $\mathscr{C} \mathscr{N}(\beta)$ by

$$
\kappa(\beta):=\left\|R^{+}(\beta)\right\|_{2}
$$

(cf. [5], [17]). By the Singular Value Decomposition,

$$
R(\beta)=U(\beta) \Sigma(\beta) V^{T}(\beta),
$$

where $U(\beta), V(\beta)$ are orthogonal matrices and

$$
\Sigma(\beta)=\operatorname{diag}\left(\sigma_{1}(\beta), \ldots, \sigma_{n}(\beta)\right)
$$

contains nonnegative diagonal elements, which we assume to be in decreasing order $\sigma_{1}(\beta) \geqslant \cdots \geqslant \sigma_{n}(\beta)$. It is clear that $\sigma_{m+1}(\beta)=\cdots=\sigma_{n}(\beta)=0$, and so we find

$$
\kappa(\beta)=\sigma_{m}^{-1}(\beta) \text {. }
$$

4. General Description of the Algorithm. With the previous results in mind, we develop a numerical method to compute solutions of conditionally stable problems. We assume that the solution has to be approximated on an interval $[0, \beta]$ within an accuracy TOL.

4.1. The Multiple Shooting Approach. The algorithm we consider is based on the ideas which were described in some detail in [14], [19]. We shall first recall the most important points here.

The integrations which are needed for multiple shooting are done adaptively for one particular solution; for the other (complementary) solutions the integrations are simply carried out on the same grid. For reasons of efficiency, a shooting interval consists of five grid points only (cf. [18]). A number of such so-called minor shooting intervals is then assembled (with the incremental recursion relations) into so-called major shooting intervals. This assembly is done in a stable way, as we first decouple the incremental recursion. For a description of the actual algorithm, however, we do not need to make the distinction between minor and major intervals. Thus, suppose we have (shooting) points $0=t_{0}, \ldots, t_{N}=\beta$. On the interval $\left[t_{i}, t_{i+1}\right]$ we typically compute a particular solution $w_{i}(t)$ and a fundamental solution $F_{i}(t)$ such that

$$
\begin{aligned}
& F_{0}\left(t_{0}\right)=Q_{0} \text { is orthogonal, } \\
& w_{i}\left(t_{i}\right)=0, \\
& F_{i-1}\left(t_{i}\right)=: F_{i}\left(t_{i}\right) U_{i}=Q_{i} U_{i},
\end{aligned}
$$


where $F_{i}\left(t_{i}\right)=Q_{i}$ is orthogonal and $U_{i}$ is upper triangular. Under conditions that often prevail, cf. [12], [19], the matrix $U_{i}$ has diagonal elements which are nonincreasing in modulus down the diagonal, reflecting the increments of the various modes of the ODE. Now since $x(t)$ can be written, for some vector $a_{i}$, as

$$
x(t)=F_{i}(t) a_{i}+w_{i}(t)
$$

we derive from the well-known multiple shooting recursion relations,

$$
F_{i+1}\left(t_{i+1}\right) a_{i+1}=F_{i+1}\left(t_{i+1}\right) U_{i} a_{i}+w_{i}\left(t_{i+1}\right) .
$$

Therefore, denoting $g_{i}:=Q_{i+1}^{T} w_{i}\left(t_{i+1}\right)$, we have

$$
a_{i+1}=U_{i} a_{i}+g_{i} \text {. }
$$

If we assume that the subspace of nonincreasing modes has rank $m$ (cf. 3.1), and introduce the partitioning

$$
\begin{gathered}
U_{i}=\left[\begin{array}{cc}
B_{i} & C_{i} \\
0 & E_{i}
\end{array}\right], \quad E_{i} \in \mathbf{R}^{m \times m}, \\
a_{i}=\left[\begin{array}{c}
a_{i}^{1} \\
a_{i}^{2}
\end{array}\right], \quad g_{i}=\left[\begin{array}{l}
g_{i}^{1} \\
g_{i}^{2}
\end{array}\right], \quad a_{i}^{2}, g_{i}^{2} \in \mathbf{R}^{m},
\end{gathered}
$$

we find the following decoupled recursion,

$$
\begin{gathered}
a_{i+1}^{2}=E_{i} a_{i}^{2}+g_{i}^{2}, \quad i=0, \ldots, N-1, \\
a_{i}^{1}=B_{i}^{-1}\left[a_{i+1}^{1}-C_{i} a_{i}^{2}-g_{i}^{1}\right], \quad i=N-1, \ldots, 0 .
\end{gathered}
$$

It is important to note that $(4.6 \mathrm{a}, \mathrm{b})$ is stable in the indicated directions. This allows us to compute a suitable particular solution and a fundamental solution (of the recursion (4.4)) in a stable way. However, we only want to have components related to the solution subspaces $\mathscr{S}_{3}$ and $\mathscr{S}_{4}$, which necessitates a special approach, to be discussed next.

4.2. Computation of Bounded Solution Components. At the point $t=\beta\left(=t_{N}\right)$ we evaluate the diagonal of $U_{N-1} \cdots U_{0}$ (implemented as a sum of logarithms of the modulus of diagonal elements with corresponding indices, to prevent overflow). If we assume that $k_{2}=0$, then we can easily find, by inspection of this product, the dimension $m$ of the subspace $\mathscr{S}_{3} \oplus \mathscr{S}_{4}$ of the nonincreasing modes. Moreover, by looking for the absolutely smallest element of the first $n-m$ diagonal elements we can find an estimate for $\lambda$ of 2.1 . This is a practical implementation of the following: Let

$$
L:=\operatorname{glb}\left(B_{N-1} \cdots B_{0}\right)
$$

where $\operatorname{glb}(M):=\min _{\|x\|=1}\|M x\|$, and define

$$
\lambda:=(\ln L) / \beta \text {. }
$$

Then the increasing modes "grow" at least like $e^{\lambda t}$. Since the desired solution $x$ may contain modes in $\mathscr{S}_{3} \oplus \mathscr{S}_{4}$, we need to compute these nonincreasing modes on $(\alpha, \beta)$ within accuracy TOL. This can be achieved by proceeding with the multiple shooting approach, outlined in Subsection 4.1, up to a further point $t=\gamma$, which is defined by

$$
\gamma:=\beta-\frac{\ln \mathrm{TOL}}{\lambda}
$$


Possible $O(1)$ components in the direction of the increasing solutions introduced at $t=\gamma$ will then have damped out by a factor TOL after backward integration from $t=\gamma$ to $t=\beta$.

Since we know the growth of the particular solution, $w_{i}^{2}\left(t_{i}\right)$, we can even use a "sharper" value for $\gamma$ if the particular solution (or the fastest growing mode in the homogeneous case) is decaying exponentially, cf. [14]. Suppose the bounded-mode subspace decays like $e^{-\mu t}$ (to be estimated as in (4.7b)), where $\mu>0$ (cf. 2.1). Then instead of $(4.7 \mathrm{c})$ we should use

$$
\gamma:=\beta-\frac{\ln \mathrm{TOL}}{\lambda-\mu} .
$$

This choice means that the relative error (in norm) at $t=\beta$ has damped by a factor TOL compared to the $O(1)$ error at $t=\gamma$. However, this certainly implies an $O$ (TOL) absolute error at $t=\beta$.

In practice, we choose shooting points as before until we reach $\gamma=: t_{M}$ (where $M>N$ ), say, and check whether

$$
\left\|B_{N}^{-1} \cdots B_{M-1}^{-1}\right\| \leqslant \text { TOL. }
$$

If this turns out to be violated, we update the value of $\gamma$ with this new increment information, until we reach a satisfactory endpoint (cf. [14]). Hence we obtain recursions like $(4.6 \mathrm{a}, \mathrm{b})$, now for $i=N, \ldots, M-1$ and $i=M-1, \ldots, N$, respectively.

We then proceed as follows: First we compute a particular solution $\left\{p_{i}\right\}_{i=0}^{M}$ of the recursion $(4.6 \mathrm{a}, \mathrm{b})$, satisfying

$$
p_{0}^{2}=0, \quad p_{M}^{1}=0 \text {. }
$$

Obviously, with this choice of $p_{M}^{1}$ we introduce an $O(1)$ truncation error, if $\left\{p_{i}\right\}_{i=0}^{M}$ is considered an approximation of $\left\{Q_{i}^{T} y_{b}\left(t_{i}\right)\right\}_{i=0}^{M}$. Since this error satisfies the homogeneous part of (4.6a) (cf. also [14]), we see that by (4.8) the truncation error at $t_{N}=\beta$ is $O$ (TOL) (and becomes even smaller for $t_{i}<t_{N}$ ). We next compute a basis $\left\{\Phi_{i}\right\}_{i=0}^{M}$ for $\mathscr{S}_{3} \oplus \mathscr{S}_{4}$ by defining

$$
\Phi_{0}^{2}=I_{m}, \quad \Phi_{M}^{1}=0 .
$$

Note that $\Phi_{i}$ is an $n \times m$ matrix, which is again computed in parts, i.e., $\left\{\Phi_{i}^{2}\right\}_{i=0}^{M}$ via the homogeneous part of (4.6a) and then $\left\{\Phi_{i}^{1}\right\}_{i=M}^{0}$ via the homogeneous part of (4.6b). $\left\{\Phi_{i}\right\}_{i=0}^{N}$, like $\left\{p_{i}\right\}_{i=0}^{N}$, is contaminated by components of increasing modes of $O$ (TOL) only. We therefore conclude that, writing

$$
a_{i}=p_{i}+\Phi_{i} c, \quad c \in \mathbf{R}^{m},
$$

the sequence $\left\{Q_{i} a_{i}\right\}_{i=0}^{N}$ is an $O(\mathrm{TOL})$ approximation of a bounded solution of (1.1). In order to determine $c$ we use the $\mathrm{BC}$

$$
\begin{aligned}
{\left[M_{0} Q_{0} \Phi_{0}+M_{1} Q_{N} \Phi_{N}\right] c=} & b-M_{0} Q_{0} p_{0}-M_{1} Q_{N} p_{N} \\
& -M_{0} w_{0}\left(t_{0}\right)-M_{1} w_{N-1}\left(t_{N}\right) .
\end{aligned}
$$

In general, this is an overdetermined system; hence, we solve it with a least-squares solver. Since we are interested in the singular values, we in fact use an SVD routine (cf. Section 6). 
5. Problems with (not Exponentially) Increasing Modes. In the previous section we assumed, for clarity of exposition, that $k_{2}=0$, that is, all increasing modes are increasing exponentially. We now wish to consider a few ways to tackle problems where $k_{2} \geqslant 1$. We shall not go into a deep analysis of existence and growth behavior of $f$ and the "bounded solution" $y_{b}$; one may consult, e.g., [5], [8]. It is important to keep in mind, however, that a slowly growing mode will, in general, force us to employ exceedingly large values of $\gamma$, cf. (4.7), unless we can indicate a special structure that enables us to obtain appropriate starting directions for much smaller “terminal points". Such a special structure is provided by an exponentially decaying source term $f(t)$. It is obvious that a backward recursion like (4.6b), now with a partitioning such that the $B_{i}$ blocks are $\left(k_{1}+k_{2}\right) \times\left(k_{1}+k_{2}\right)$, should work satisfactorily; that is, if the point $\gamma$ is chosen such that

$$
\frac{\|f(\gamma)\|}{\|f(\beta)\|}\left\|B_{M-1}^{-1} \cdots B_{N}^{-1}\right\| \leqslant \text { TOL, }
$$

we should expect the error due to this "truncation" to be bounded by TOL. Hence, in the sequel we shall assume that at least one of the components of $f$ behaves like a rational function in $t$ (generalizations including fractional powers of $t$ are again obvious).

A central point in our discussion is played by the fact that we can (easily) compute the "direction" of the subspace $\mathscr{S}_{2}$ : By this we mean that we can find a basis within an accuracy TOL using the multiple shooting approach of Subsection 4.1. We first extend our interval of integration to a point $\delta=t_{S}$, say, as follows: Instead of computing complete fundamental solutions we only compute the first $k_{1}+k_{2}$ columns. On $\left[t_{i}, t_{i+1}\right]$ we thus have an initial value $Q_{i}^{1}$, where $Q_{i}^{1}$ is an $n \times\left(k_{1}+k_{2}\right)$ matrix consisting of orthonormal columns. Instead of choosing $w_{i+1}\left(t_{i+1}\right)=0$, we take the projection

$$
w_{i+1}\left(t_{i+1}\right)=\left[I-Q_{i+1}^{1}\left[Q_{i+1}^{1}\right]^{T}\right] w_{i}\left(t_{i+1}\right)
$$

Write

$$
x\left(t_{i}\right)=Q_{i}^{1} \hat{a}_{i}+w\left(t_{i}\right), \quad \hat{a}_{i} \in \mathbf{R}^{k_{1}+k_{2}} .
$$

This computation, which is in fact the stabilized march (cf. [16], [19]), gives, after matching, a recursion like $(4.6 \mathrm{a})$,

$$
\hat{a}_{i}=\hat{B}_{i}^{-1}\left[\hat{a}_{i+1}-\hat{h}_{i}\right]
$$

with

$$
\hat{h}_{i}=\left[Q_{i+1}^{1}\right]^{T}\left[w_{i}\left(t_{i+1}\right)-w_{i+1}\left(t_{i+1}\right)\right]=\left[Q_{i+1}^{1}\right]^{T} w_{i}\left(t_{i+1}\right) .
$$

If we partition $\hat{B}_{i}$ as

$$
\hat{B}_{i}=\left[\begin{array}{cc}
B_{i} & * \\
0 & *
\end{array}\right], \quad B_{i} \in \mathbf{R}^{k_{1} \times k_{1}},
$$

then the point $\delta=t_{S}$ is chosen such that

$$
\left\|B_{M}^{-1} \cdots B_{S-1}^{-1}\right\| \approx \text { TOL. }
$$

Now let $\left\{\Psi_{i}\right\}_{i=0}^{S}$ be a matrix solution of the homogeneous part of (5.3a), with

$$
\Psi_{S}=\left[\begin{array}{c}
0 \\
I_{k_{2}}
\end{array}\right]
$$


( $\Psi_{i}$ thus being a $\left(k_{1}+k_{2}\right) \times k_{2}$ matrix). Then $\operatorname{span}\left(\Psi_{i}\right)$, for $i \leqslant M$, represents the directions of the solutions in $\mathscr{S}_{2}$ at $t=t_{i}$ within an accuracy of TOL. Similarly, by defining

$$
q_{s}=0
$$

we can compute a particular solution $\left\{q_{i}\right\}_{i=0}^{S}$ of (5.3a). With the vector $\hat{c} \in \mathbf{R}^{k_{2}}$, and

$$
\hat{a}_{i}=q_{i}+\Psi_{i} \hat{c},
$$

we can thus find via (5.2) any solution in $\mathscr{S}_{2} \oplus y_{b}$ within an accuracy of TOL for $t \leqslant \gamma$.

The problem now is to "remove" the $\mathscr{S}_{2}$ components from a suitable solution. To that end, factorize $\Psi_{i}$ as a matrix with orthonormal columns $X_{i}$ and an upper triangular matrix $R_{i}$,

$$
\Psi_{i}=X_{i} R_{i}
$$

Then define the orthogonal projection

$$
Z_{i}=X_{i} X_{i}^{T}
$$

(a $\left(k_{1}+k_{2}\right) \times\left(k_{1}+k_{2}\right)$ matrix). We now consider the following two inhomogeneous recursions,

$$
\begin{aligned}
\hat{r}_{i} & =\hat{B}_{i}^{-1}\left[\hat{r}_{i+1}-Z_{i+1} \hat{h}_{i}\right], \\
\hat{s}_{i} & =\hat{B}_{i}^{-1}\left[\hat{s}_{i+1}-\left[I-Z_{i+1}\right] \hat{h}_{i}\right] .
\end{aligned}
$$

Clearly, $\left\{\hat{r}_{i}+\hat{s}_{i}\right\}$ satisfies (5.3a). Although the recursions (5.3a) and (5.8a,b) were defined for $i=M, \ldots, S-1$ only, we may consider them for $i=1, \ldots, M-1$ as well, since we can repartition (4.4) in a straightforward way with $\hat{B}_{i}$ being the left upper part of $U_{i}$.

If we use (5.8a) with $\hat{r}_{S}=0$ we find

$$
\hat{r}_{i}=-\sum_{j=i}^{S-1} \hat{B}_{i}^{-1} \cdots \hat{B}_{j}^{-1} Z_{j+1} \hat{h}_{j},
$$

whence $\hat{r}_{i} \in \operatorname{span}\left(Z_{i}\right)=\operatorname{span}\left(\Psi_{i}\right)$.

Similarly, if we use $(5.8 \mathrm{~b})$ with $\hat{s}_{S}=0$, we find

$$
\hat{s}_{i}=-\sum_{j=i}^{S-1} \hat{B}_{i}^{-1} \cdots \hat{B}_{j}^{-1}\left(I-Z_{j+1}\right) \hat{h}_{j},
$$

so $\hat{s}_{i} \in \operatorname{span}\left(I-Z_{i}\right)$. If we write for ease of notation the transformed bounded solution $y_{b}$ as

$$
\hat{u}_{i}:=Q_{i}^{T} y_{b}\left(t_{i}\right),
$$

then we seek to determine $\hat{a}_{i}$ of (5.3a) such that in

$$
\hat{a}_{i}=\hat{u}_{i}+\Psi_{i} \hat{c}
$$

the norm of $\hat{c}$ is as small as possible. From (5.9b) we immediately see that

$$
\hat{s}_{i}=\left(I-Z_{i}\right) \hat{u}_{i} .
$$


This means that it is possible to obtain the component of $y_{b}(t)$ in the orthogonal complement of $\operatorname{span}\left(Y(t) P_{2}\right)$ (in $\operatorname{span}\left(Y(t) P_{1}\right) \oplus \operatorname{span}\left(Y(t) P_{2}\right)$ ) for values $t \leqslant \gamma$, and within an accuracy of TOL, in a stable way (note that (5.8b) is stable). The component of $y_{b}(t)$ in the orthogonal complement of $\operatorname{span}\left(Y(t) P_{1}\right) \oplus \operatorname{span}\left(Y(t) P_{2}\right)$ is simply found from (4.6b). It remains to compute the component in $\operatorname{span}\left(Y(t) P_{2}\right)$. (Of course, spaces here have a strict geometrical, not a functional meaning!)

In order to compute the latter component we first remark that both the polynomial growth of solutions in $\mathscr{S}_{2}$ and the rational growth of $f$ make it quite reasonable to expect that $\hat{s}_{0}=O\left(\left(1 / t_{S}\right)^{p}\right)$ for some integer $p>0$. To simplify further discussion, we therefore make the following

Assumption 5.13. Let $\left\{\hat{s}_{i}(j)\right\}_{i=0}^{j}$ satisfy $(5.8 \mathrm{~b})$ and $\hat{s}_{j}(j)=0$. Define $\hat{s}_{0}^{*}(=$ $\left.\left(I-Z_{0}\right) \hat{u}_{0}\right)=\lim _{j \rightarrow \infty} \hat{s}_{0}(j)$ and the vectors $d(j) \in \mathbf{R}^{k_{2}}$ by $\hat{s}_{0}(j)=\Psi_{0} \hat{d}(j)$. Now assume that $\hat{d}(j)=\left(\delta_{1}(j), \ldots, \delta_{k_{2}}(j)\right)^{T}$ has an asymptotic expansion, as $j \rightarrow \infty$, of the form

$$
\delta_{l}(j)=\zeta_{l, 0}+\zeta_{l, 1}\left(\frac{1}{t_{j}+1}\right)^{\omega}+\zeta_{l, 2}\left(\frac{1}{t_{j}+1}\right)^{2 \omega}+\cdots, \quad \omega \in \mathbf{R}_{+} .
$$

The solution $\left\{\hat{s}_{i}^{*}\right\}=\left\{\left(I-Z_{i}\right) \hat{u}_{i}\right\}$ is then approximated as follows. Choose points $t_{j}$, say $\beta, \gamma, \delta$, and compute $\hat{s}_{0}(j)$. By Richardson extrapolation we can now find, approximately, the values of $\zeta_{l, 0}$. If implemented properly, one can even estimate the accuracy of the approximation; of course, TOL acts again as the bound for maximal obtainable accuracy, so this should determine the order of the extrapolation scheme. Once we have $\left(\zeta_{1,0}, \ldots, \zeta_{k_{2}, 0}\right)^{T}=: \hat{d}^{*}\left(=\lim _{j \rightarrow \infty} \hat{d}(j)\right)$, we find $\hat{s}_{0}^{*}$ from

$$
\hat{s}_{0}^{*}=\Psi_{0} \hat{d}^{*}
$$

The other iterates $\hat{s}_{i}^{*}$ can now be found fairly easily. We have for some vector $\hat{c}(N) \in \mathbf{R}^{k_{2}}$,

$$
0=\hat{s}_{N}(N)=\hat{s}_{N}^{*}+\Psi_{N} \hat{c}(N)
$$

so that

$$
\hat{s}_{0}(N)=\hat{s}_{0}^{*}+\Psi_{0} \hat{c}(N) .
$$

Since $\hat{s}_{0}(N), \hat{s}_{0}^{*}$ and $\Psi_{0}$ are known, we can thus find the vector $\hat{c}(N)$ from $(5.15 \mathrm{~b})$. Note that certainly the last $k_{2}$ rows of $\Psi_{0}$ define a nonsingular matrix. Substitution of this vector in (5.15a) yields $\hat{s}_{N}^{*}$ and hence via (5.8b) the entire sequence $\left\{\hat{s}_{i}^{*}\right\}_{i=0}^{N}$. It is worth noting that the "slow" growth of solutions in $\mathscr{S}_{2}$ not only makes extrapolation meaningful but also the computation of the vector $\hat{c}(N)$ in $(5.15 \mathrm{~b})$ fairly stable. For this, observe that $(5.15 \mathrm{~b})$ is overdetermined. Therefore, we only need the last $k_{2}$ rows of $\Psi_{0}$, which form an upper triangular matrix (which is not unduly small in norm!)

Thus far, we only considered the question of how to "remove" $\mathscr{S}_{2}$ modes from a bounded particular solution. Our discussion was based on a detailed discussion of the inhomogeneous shooting recursion (5.3a). This means that we can utilize the same derivations for obtaining modes in $\mathscr{S}_{2}$ which are approximated within accuracy TOL. Indeed, by partitioning the matrices $U_{i}$ as

$$
U_{i}=\left[\begin{array}{cc}
\hat{B}_{i} & \hat{C}_{i} \\
0 & \hat{E}_{i}
\end{array}\right]
$$


with $\hat{B}_{i}$ of order $k_{1}+k_{2}$, the backward recursion (4.6b) (with corresponding partitioning) can be identified with (5.3a), with special vectors $\hat{h}_{i}^{1}$ for each such mode. We remark that this is only needed for not exponentially decaying modes (as we noted for "bounded" solutions which are exponentially decaying). For solutions in $\mathscr{S}_{4}$ we just have to choose $\gamma\left(\right.$ or $\delta$ ) large enough such that $\left\|E_{M-1} \cdots E_{N}\right\| \leqslant$ TOL.

Although we believe that Assumption 5.13 is realistic (and certainly covers cases discussed in [8]), we may alternatively consider problems where $y_{b}(t)$ tends to a limit but the solutions in $\mathscr{S}_{2}$ do not necessarily. We assume that $y_{b}(t)$ has an asymptotic expansion in powers of $(t+1)^{-1}$ and that solutions in $\mathscr{S}_{2}$ grow polynomially. Then denote for the solution $\left\{q_{j}\right\}$ (cf. (5.3a), (5.5))

$$
p_{i}:=Q_{i}^{1} q_{i}
$$

For $S-i$ large enough, $p_{i}$ can be written as

$$
p_{i}=y_{b}\left(t_{i}\right)+Q_{i}^{1} \Psi_{i} \hat{c}
$$

where $\hat{c} \in \mathbf{R}^{k_{2}}$ (cf. (5.6)). Now assume that $y_{b}(t)$ has an asymptotic expansion as $t \rightarrow \infty$,

$$
y_{b}(t)=r_{0}+r_{1}\left(\frac{1}{t+1}\right)^{\omega}+r_{2}\left(\frac{1}{t+1}\right)^{2 \omega}+\cdots, \quad \omega \in \mathbf{R}_{+}
$$

(where $r_{i} \in \mathbf{R}^{n}$ ). If $t_{S}$ is large enough we can monitor $p_{i}$ at several places and estimate $r_{0}, r_{1}, \ldots$ and the vector $\hat{c}$ by solving a (possibly overdetermined) linear system. By substituting these values of $r_{0}, r_{1}, \ldots$ into the expansion for $y_{b}\left(t_{S}\right)$, we can even refine this estimate by using the backward recursion (5.3a) with "initial" value $\left[Q_{S}^{1}\right]^{T} y_{b}\left(t_{S}\right)$ : This should give an even more "pure" $y_{b}\left(t_{0}\right)$, providing a more accurate estimate of $\hat{c}$ via (5.16b), and hence of $y_{b}$.

6. Choice of Partitioning; Underdetermined Problems. The last question to be considered is how one should deal with nongrowing/nondecaying components and modes, that is, whether or not they should be controlled by an initial condition (cf. Property 2.8). To a certain extent the partitioning of the nongrowing/nondecaying solution subspace into parts that belong to $\mathscr{S}_{2}$ and $\mathscr{S}_{3}$, respectively, is arbitrary. We now opt for a choice where $k_{3}$ is maximal. This may make (4.12) a rank-deficient system.

One of the advantages of the singular value decomposition is that we can easily use it to compute the minimal norm solution of such a rank-deficient system. If we write

$$
\tilde{R}_{N}=M_{0} Q_{0} \Phi_{0}+M_{1} Q_{N} \Phi_{N}
$$

(an approximation of $O$ (TOL) to $R(\beta)$, cf. (3.3b)), and

$$
\tilde{R}_{N}=\tilde{U}_{N} \tilde{\Sigma}_{N} \tilde{V}_{N}^{T}
$$

with $\tilde{R}_{N}, \tilde{V}_{N}$ orthogonal and

$$
\tilde{\Sigma}_{N}=\operatorname{diag}\left(\sigma_{1}(N), \ldots, \sigma_{m}(N)\right), \quad \sigma_{1}(N) \geqslant \cdots \geqslant \sigma_{m}(N)
$$

(cf. (3.6a, b)), we can first determine the numerical rank of (4.12). As before, we treat quantities smaller than TOL as numerically zero. If $\sigma_{l+1}(N), \ldots, \sigma_{m}(N) \leqslant$ TOL, we define

$$
\tilde{\Sigma}_{N}^{+}=\operatorname{diag}\left(\sigma_{1}^{-1}(N), \ldots, \sigma_{l}^{-1}(N), 0, \ldots, 0\right)
$$


and, otherwise,

$$
\tilde{\Sigma}_{N}^{+}=\operatorname{diag}\left(\sigma_{1}^{-1}(N), \ldots, \sigma_{m}^{-1}(N)\right) .
$$

We thus find a pseudo-inverse of $\tilde{R}_{N}$,

$$
\tilde{R}_{N}^{+}=\tilde{V}_{N} \tilde{\Sigma}_{N}^{+} U_{N}^{T}
$$

This matrix $\tilde{R}_{N}^{+}$determines a vector $c$ via (4.12) and hence a sequence $\left\{a_{i}\right\}$ via (4.11), and thus, finally, a particular solution $x$ via (4.2). If $l=m$, this solution is an approximation to "the" unique bounded solution. The condition number of the BVP is then found to be $\sigma_{m}^{-1}(N)$ (cf. (3.7)). If $\tilde{R}_{N}$ is rank-deficient we can still give some potentially meaningful results, although we have no uniqueness. Indeed, in addition to the solution $x$ found as before, we can also give basis solutions of the complementary solution space, so that we actually characterize the $(m-l)$-dimensional manifold of solutions solving the BVP. To see this, let

$$
\tilde{V}_{N}:=\left(v_{N}(1)|\cdots| v_{N}(m)\right) \text {. }
$$

Then it follows from the construction of $\Phi_{i}$ and $R_{N}$ that

$$
y\left(t_{i}, j\right):=Q_{i} \Phi_{i} v_{N}(j), \quad j=l+1, \ldots, m,
$$

defines a solution $\left\{y\left(t_{i}, j\right)\right\}_{i=1}^{N}$ ( $j$ fixed) which satisfies a BVP with a BC like (1.2), but with homogeneous BC's. Hence the general solution of the rank-deficient case at the point $t_{i}$ is given by

$$
x\left(t_{i}\right)+\sum_{j=l+1}^{m} \varepsilon_{j} y\left(t_{i}, j\right), \quad \varepsilon_{j} \in \mathbf{R} .
$$

For such a problem a meaningful condition number is given by $\sigma_{l}^{-1}(N)$, unless all singular values are zero.

7. Numerical Examples. In this section we give a number of examples to illustrate the previously outlined algorithms.

Example 7.1. Consider the ODE

$$
\frac{d x}{d t}=\left[\begin{array}{cc}
10 \cos 2 t & -1+10 \sin 2 t \\
1+10 \sin 2 t & -10 \cos 2 t
\end{array}\right] x+e^{-t}\left[\begin{array}{c}
-10(\cos 2 t+\sin 2 t) \\
-2+10(\cos 2 t-\sin 2 t)
\end{array}\right]
$$

A bounded particular solution $y_{b}$ is given by

$$
y_{b}(t)=e^{-t}\left[\begin{array}{l}
1 \\
1
\end{array}\right]
$$

and a fundamental solution $F$ by

$$
F(t)=\left[\begin{array}{rr}
\cos t & -\sin t \\
\sin t & \cos t
\end{array}\right] \operatorname{diag}\left(e^{10 t}, e^{-10 t}\right) .
$$

As BC's we use

$$
\left[\begin{array}{ll}
1 & 0 \\
0 & 1
\end{array}\right] x(0)=\left[\begin{array}{l}
1 \\
2
\end{array}\right]
$$

so that

$$
x=e^{-t}\left[\begin{array}{l}
1 \\
1
\end{array}\right]+e^{-10 t}\left[\begin{array}{r}
-\sin t \\
\cos t
\end{array}\right]
$$


TABLE 7.1

Numerical results for Example 7.1

\begin{tabular}{|c|c|c|}
\hline$t$ & $x$ exact & abs. error \\
\hline 0 & $\begin{array}{l}.100+1 \\
.200+1\end{array}$ & $\begin{array}{l}.44-7 \\
.22-14\end{array}$ \\
\hline 1 & $\begin{array}{l}.368+0 \\
.368+0\end{array}$ & $\begin{array}{l}.19-8 \\
.29-8\end{array}$ \\
\hline 2 & $\begin{array}{l}.135+0 \\
.135+0\end{array}$ & $\begin{array}{l}.43-8 \\
.11-8\end{array}$ \\
\hline 3 & $\begin{array}{l}.489-1 \\
.489-1\end{array}$ & $\begin{array}{l}.12-8 \\
.20-8\end{array}$ \\
\hline 4 & $\begin{array}{l}.183-1 \\
.183-1\end{array}$ & $\begin{array}{l}.20-8 \\
.20-8\end{array}$ \\
\hline 5 & $\begin{array}{l}.674-2 \\
.674-2\end{array}$ & $\begin{array}{l}.20-8 \\
.31-8\end{array}$ \\
\hline 6 & $\begin{array}{l}.248--2 \\
.248-2\end{array}$ & $\begin{array}{l}.11-9 \\
.27-8\end{array}$ \\
\hline 7 & $\begin{array}{l}.912-3 \\
.912-3\end{array}$ & $\begin{array}{l}.19-8 \\
.92-9\end{array}$ \\
\hline 8 & $\begin{array}{l}.335-3 \\
.335-3\end{array}$ & $\begin{array}{l}.13-8 \\
.83-9\end{array}$ \\
\hline 9 & $\begin{array}{l}.123-3 \\
.123-3\end{array}$ & $\begin{array}{l}.42-9 \\
.20-8\end{array}$ \\
\hline 10 & $\begin{array}{l}.454-4 \\
.454-4\end{array}$ & $\begin{array}{l}.12-8 \\
.59-9\end{array}$ \\
\hline
\end{tabular}

In Table 7.1 we list the values of $x$ and associated errors resulting from a computation with TOL $=10^{-6}, \beta=10$. The algorithm found

$$
\gamma=11.38\left(e^{10(\gamma-\beta)} \approx \mathrm{TOL}^{-1}\right)
$$

the condition number $\kappa$ was found to be $\approx 1$.

Example 7.4. We carried out an experiment similar to Example 7.1, but now with a BC

$$
\left[\begin{array}{ll}
1 & 0 \\
1 & 0
\end{array}\right] x(0)=\left[\begin{array}{l}
1 \\
1
\end{array}\right]
$$

As can be seen quite easily, the row vectors of this $\mathrm{BC}$ are orthogonal to the initial value of the decaying mode, so we expect no unique bounded solution. The algorithm nevertheless gave a bounded particular solution and a basis for the stable mode space. From Table 7.2 we see that we might construct the same solution as in Example 7.1.

Example 7.6. Written as a first-order system, a one-dimensional Schrödinger equation with potential given in [3], can be written as

$$
\frac{d x}{d t}=\left[\begin{array}{cc}
0 & 20 \tanh ^{2}(t)-11 \\
1 & 0
\end{array}\right] x
$$


TABLE 7.2

Numerical results for Example 7.4

\begin{tabular}{|c|c|c|}
\hline$t$ & part. sol'n & stable sol'n \\
\hline 0 & $.100+1$ & $-.294-7$ \\
& $.100+1$ & $-.707+0$ \\
1 & $.368+0$ & $.270-4$ \\
& $.368+0$ & $-.174-4$ \\
2 & $.135+0$ & $.132-8$ \\
& $.135+0$ & $.607-9$ \\
. &. &. \\
. &. &. \\
. &. &. \\
. &. & $.136-43$ \\
10 & $.454-4$ & $.209-43$ \\
\hline
\end{tabular}

Actually, this is an eigenvalue problem whose first eigenvalue is equal to 11 . Consider the BC's

$$
\left[\begin{array}{ll}
1 & 0 \\
0 & 0
\end{array}\right] x(0)+\left[\begin{array}{ll}
0 & 0 \\
1 & 0
\end{array}\right] x(\infty)=\left[\begin{array}{l}
0 \\
0
\end{array}\right]
$$

Clearly, we do not have a unique solution. We asked for output on $[0,4]$ with TOL $=10^{-6}$. The algorithm computed a terminal value $\gamma=5.96$. One should realize that the "unstable" solutions grow like $e^{3 t}$ and the desired "bounded" solution like $e^{-3 t}$. Hence at $t=\gamma$ we have the theoretical point where their relative growth equals $\mathrm{TOL}^{-1}$, and this is quite close to the value of $\gamma$ actually found (cf. (4.7c)). The results of the algorithm are displayed in Table 7.3. The last column contains the absolute error after normalization of the "stable" solution (by comparing the initial values to the exact solution). Note that the absolute errors decay with the magnitude of $x$.

TABLE 7.3

Numerical results for Example 7.6

\begin{tabular}{|c|c|c|c|}
\hline$t$ & stable sol'n & exact sol'n & abs. error \\
\hline 0 & $-.105-6$ & $.000+0$ & $.10-6$ \\
& $-.100+1$ & $+.100+1$ & $.25-6$ \\
.8 & $-.278+0$ & $+.278+0$ & $.17-6$ \\
& $+.320+0$ & $-.320+0$ & $.24-6$ \\
1.6 & $-.538-1$ & $+.538-1$ & $.40-7$ \\
& $+.140+0$ & $-.140+0$ & $.84-7$ \\
2.4 & $-.573-2$ & $+.573-2$ & $.23-9$ \\
& $+.167-1$ & $-.167-1$ & $.53-9$ \\
3.2 & $-.537-3$ & $+.537-3$ & $.47-9$ \\
& $+.160-2$ & $-.160-2$ & $.37-10$ \\
4.0 & $-.490-4$ & $+.490-4$ & $.29-9$ \\
& $+.147-3$ & $-.147-3$ & $.10-8$ \\
\hline
\end{tabular}


Example 7.9. Consider the ODE

$$
\frac{d x}{d t}=\left[\begin{array}{cc}
10 & 0 \\
0 & 0
\end{array}\right] x+f(t), \quad t \geqslant 0
$$

where

$$
f(t)=\left(-\frac{1}{(t+1)^{2}}-\frac{2}{(2 t+1)^{2}}-\frac{10}{t+1}-\frac{10}{2 t+1},-\frac{1}{(t+1)^{2}}-\frac{2}{(2 t+1)^{2}}\right)^{T} .
$$

A particular solution is given by

$$
x(t)=\frac{3 t+2}{(t+1)(2 t+1)}\left[\begin{array}{l}
1 \\
1
\end{array}\right] .
$$

As BC's we take

$$
\left[\begin{array}{ll}
1 & 0 \\
0 & 0
\end{array}\right] x(0)=\left[\begin{array}{l}
2 \\
0
\end{array}\right]
$$

Suppose we want a solution on $[0,10]$ with TOL $=10^{-4}$. Because the homogeneous part of (7.10) has a constant solution and $x$ is decaying only moderately, we use extrapolation. Anticipating $\omega=1$ in the expansion (cf. Assumption 5.13), we used Richardson extrapolation for $t=10,20$, and 30 . We obtained values for $\hat{s}_{0}$ equal to 2.13851, 2.07202, 2.04865, respectively. After two extrapolation steps we found

$$
\hat{s}_{0}^{*} \sim\left[\begin{array}{c}
0 \\
2.00016
\end{array}\right]
$$

which gave us an approximation for $x(0)$ with an error of $1.6 \times 10^{-4}$. Note that we would have been forced to go as far as $t \approx 10^{4}$ to obtain a similar accuracy for the "truncation error", without extrapolation!

Example 7.14. Consider the ODE

$$
\frac{d x}{d t}=\left[\begin{array}{rr}
-\sin t+10 \cos t & \cos t+\sin t \\
-\cos t-10 \sin t & -\sin t+\cos t
\end{array}\right] x+f(t), \quad t \geqslant 0
$$

where

$$
f(t)=-\frac{1}{1+t}\left[\begin{array}{c}
\frac{1}{1+t}+10 \cos t \\
\frac{1}{1+t}-10 \sin t
\end{array}\right]
$$

Consider the BC's

$$
x(0)=\left[\begin{array}{l}
2 \\
2
\end{array}\right]
$$

A particular (bounded) solution is given by

$$
y_{b}(t)=\frac{1}{t+1}\left[\begin{array}{l}
1 \\
1
\end{array}\right]
$$

and a fundamental system by

$$
F(t)=\left[\begin{array}{rr}
e^{10 t} \cos t & \sin t \\
-e^{10 t} \sin t & \cos t
\end{array}\right]
$$


Since $F(t)$ does not have asymptotically constant directions, Assumption 5.13 is not applicable. Nevertheless, $y_{b}$ has an asymptotic expansion. We computed "trial" values at $t_{i}=10,20,30$, starting at $t=32$, and set $\gamma_{2}=\gamma_{3}=\cdots=0$. We obtained the following values, see Table 7.4, using a tolerance TOL $=10^{-4}$ (cf. (5.16)).

TABLE 7.4

Numerical results for Example 7.14

\begin{tabular}{|c|c|}
\hline$t_{i}$ & $p_{i}$ \\
\hline 30 & -.0092286 \\
& .038734 \\
\hline 20 & .0085953 \\
& .064754 \\
\hline 10 & -.1375201 \\
& .055678 \\
\hline
\end{tabular}

We found (cf. (5.17))

$$
r_{0}=\left[\begin{array}{ll}
.12 & -4 \\
.59 & -4
\end{array}\right], \quad r_{1}=\left[\begin{array}{ll}
1.0 & +1 \\
1.0 & +1
\end{array}\right], \quad \hat{c}=.4199 \times 10^{-1}
$$

(actually the coefficients in $r_{1}$ were accurate up to five decimals).

Acknowledgment. The author would like to thank R. England and the referee for helpful comments regarding this paper.

Department of Mathematics

Catholic University

Toernooiveld

6525 ED Nijmegen, The Netherlands

1. A. BAYLISS, "A double shooting scheme for certain unstable and singular boundary value problems," Math. Comp., v. 32, 1978, pp. 61-71.

2. W. A. COPPEL, Dichotomies in Stability Theory, Lecture Notes in Math., vol. 629, Springer-Verlag, Berlin and New York, 1978.

3. R. England, A Program for the Solution of Boundary Value Problems for Systems of Ordinary Differential Equations, Culham Laboratory Report, PDN 3/73, 1976.

4. F. R. DE Hoog \& R. M. M. MattheiJ, “On dichotomy and well-conditioning in BVP," SIAM J. Numer. Anal., 1987. (To appear.)

5. F. R. DE Hoog \& R. WeISS, "An approximation theory for boundary value problems on infinite intervals," Computing, v. 24, 1980, pp. 227-239.

6. F. R. DE HOOG \& R. WEISS, "On the boundary value problem for systems of ordinary differential equations with a singularity of the second kind," SIAMJ. Math. Anal., v. 11, 1980, pp. 41-60.

7. W. F. LANGFORD, "A shooting algorithm for the best least squares solution of two-point boundary value problems,” SIAM J. Numer. Anal., v. 14, 1977, pp. 527-542.

8. M. LeNTINI \& H. B. KelleR, "Boundary value problems on semi-infinite intervals and their numerical solution," SIAM J. Numer. Anal., v. 17, 1980, pp. 577-604.

9. P. MARKOWICH, "A theory for the approximation of solutions of boundary value problems on infinite intervals," SIAM J. Math. Anal., v. 13, 1982, pp. 484-513.

10. J. L. MaSSERA \& J. J. SChäfFER, Linear Differential Equations and Function Spaces, Academic Press, New York, 1966. 
11. R. M. M. MATTHEIJ, "On approximating smooth solutions of linear singularly perturbed ODE," in Numerical Analysis of Singular Perturbation Problems (P. W. Hemker \& J. J. H. Miller, eds.), Academic Press, London, 1979, pp. 457-465.

12. R. M. M. MATTHEIJ, "Characterizations of dominant and dominated solutions of linear recursions," Numer. Math., v. 35, 1980, pp. 421-442.

13. R. M. M. MATTHEIJ, "Estimates for the errors in the solution of linear boundary value problems due to perturbations," Computing, v. 27, 1981, pp. 299-318.

14. R. M. M. MatTheIJ, "Stable computation of solutions of unstable linear initial value recursions," $B I T$, v. 22, 1982, pp. 79-93.

15. R. M. M. MATTHEIJ, "The conditioning of linear boundary value problems," SIAM J. Numer. Anal., v. 19, 1982, pp. 963-978.

16. R. M. M. MATTHEIJ, "Decoupling and stability of algorithms for boundary value problems," SIAM Rev., v. 27, 1985, pp. 1-44.

17. R. M. M. MatTheiJ \& F. R. DE Hoog, "On non-invertible boundary value problems," Proceedings of the Workshop on Numerical Boununry Value ODE's (U. Ascher \& R. D. Russell, eds.), Birkhäuser, Boston, 1985, pp. 55-75.

18. R. M. M. MattheiJ \& G. W. M. StaARinK, “On optimal shooting intervals," Math. Comp., v. 42, 1984, pp. 25-40.

19. R. M. M. Mattheij \& G. W. M. STaArink, "An efficient algorithm for solving general linear two point BVP," SIAM J. Sci. Statist. Comput., v. 5, 1984, pp. 745-763.

20. T. N. Robertson, “The linear two-point boundary value problem on an infinite interval," Math. Comp., v. 25, 1971, pp. 475-481.

21. G. SöDERLind \& R. M. M. MatrheIJ, "Stability and asymptotic estimates in nonautonomous linear differential systems," SIAM J. Math. Anal., v. 16, 1985, pp. 69-92.

22. G. W. STEWART, "On the perturbation of pseudo-inverses, projections and linear least squares problems,” SIAM Rev., v. 19, 1977, pp. 634-662. 